\title{
Where have all the black holes gone?
}

\author{
T. Beckert ${ }^{1}$ and W. J. Duschl ${ }^{2,1}$ \\ 1 Max-Planck-Institut für Radioastronomie, Auf dem Hügel 69, 53121 Bonn, Germany \\ 2 Institut für Theoretische Astrophysik, Tiergartenstraße 15, 69121 Heidelberg, Germany
}

Received 24 April 2001 / Accepted 18 March 2002

\begin{abstract}
We have calculated stationary models for accretion disks around super-massive black holes in galactic nuclei. Our models show that below a critical mass flow rate of $\sim 3 \times 10^{-3} \dot{M}_{\text {Edd }}$ advection will dominate the energy budget while above that rate all the viscously liberated energy is radiated. The radiation efficiency declines steeply below that critical rate. This leads to a clear dichotomy between AGN and normal galaxies which is not so much given by differences in the mass flow rate as by the radiation efficiency. At very low mass accretion rates below $5 \times 10^{-5} \dot{M}_{\text {Edd }}$ synchrotron emission and Bremsstrahlung dominate the SED, while above $2 \times 10^{-4} \dot{M}_{\text {Edd }}$ the inverse Compton radiation from synchrotron seed photons produce flat to inverted SEDs from the radio to X-rays. Finally we discuss the implications of these findings for AGN duty cycles and the long-term AGN evolution.
\end{abstract}

Key words. accretion, accretion disks - black hole physics - radiation mechanisms: non-thermal - galaxies: active galaxies: nuclei

\section{Evidence for black holes and advection-dominated accretion}

The existence of black holes $(\mathrm{BH})$ in the centers of galaxies is now widely accepted and the best mass determinations are known for $\mathrm{Sgr} \mathrm{A}^{*}$ in the Galactic Center $\left(M_{\mathrm{BH}}=2.6 \times 10^{6} M_{\odot}\right)$ from the stellar velocity dispersion, and for NGC 4258 from Keplerian rotation of maser spots in an accretion disk. Beside these very low luminosity AGNs, the masses of $\mathrm{BH}$ in quasars have been estimated from their continuum luminosity and the $\mathrm{H} \beta$ line width (Laor 2000). It turns out, that radio-loud quasars and radio galaxies, which show powerful jets, are found in large elliptical galaxies with the most massive $\mathrm{BHs} \sim 10^{9} M_{\odot}$.

Seyfert Galaxies, on the other hand, are spiral galaxies which host an AGN. Activity in the nucleus, which is powered by accretion into a $\mathrm{BH}$, can be discriminated against starbursts in Seyferts from radio and X-ray observations. Radio cores and jets with brightness temperatures above $10^{8} \mathrm{~K}$ have been detected in some Seyferts (Ulvestad et al. 1999; Mundell et al. 2000; Falcke et al. 2000). Their flux stability over several years exculdes radio supernovae as the power supply. The X-ray emission shows rapid variability and in some cases a redshifted $\mathrm{Fe}$ $\mathrm{K} \alpha$ line, which is an indicator of relativistic motion in the accretion disk around the $\mathrm{BH}$. The masses of $\mathrm{BH}$ in some Seyfert 1 galaxies have been measured by reverberation mapping of variable and correlated continuum and line

Send offprint requests to: T. Beckert, e-mail: tbeckert@mpifr-bonn.mge.de emission (Peterson \& Wandel 2000). These measurements are in reasonable agreement with the $M_{\mathrm{BH}}-\sigma$ relation of enclosed mass $\left(\sim M_{\mathrm{BH}}\right)$ versus velocity dispersions $\sigma$ in bulges of normal galaxies (Gebhardt et al. 2000). It is therefore reasonable to assume the existence of supermassive $\mathrm{BH}$ in most elliptical galaxies and spirals with bulges.

While in the high luminosity objects both jet and accretion disk can be identified in the spectrum, the situation is different in less luminous AGNs like weak Seyfert Galaxies and LINERs. But even here small scale jets are commonly found (Falcke et al. 2000) and argue for the existence of BHs. For instance NGC 4258 is an interesting transition object showing both an outer irradiated thin accretion disk and a small scale radio jet. A geometrically thin standard accretion disk close to the black hole can not be identified, but the ionizing X-rays maybe produced at the base of the jet, which can be identical with the proposed advection-dominated accretion flow (ADAF) within $100 R_{\mathrm{S}}$ (Gammie et al. 1999; $R_{\mathrm{S}}$ : Schwarzschild radius). At even lower luminosities the Galactic Center $\left(\mathrm{Sgr} \mathrm{A}^{*}\right)$ with a BH mass of $2.6 \times 10^{6} M_{\odot}$ (Genzel et al. 1997 ) is the only visible AGN with a power output of $\approx 2 \times 10^{-10} L_{\text {Edd. }}$. A comparable object in any other galaxy (spiral or elliptical) would not be seen as an AGN. Assuming an spherical and adiabatic Bondi inflow, cooled only by Bremsstrahlung, gives an radiation efficiency $\eta=$ $9 \times 10^{-3}\left(L / L_{\mathrm{Edd}}\right)$ (Frank et al. 1992). Bremsstrahlung will be emitted in X-rays and the Chandra detection (Baganoff et al. 2001) of $L_{\mathrm{X}} \sim 2 \times 10^{33} \mathrm{erg} \mathrm{s}^{-1}$ is consistent with a mass accretion rate of $\dot{M}=1.6 \times 10^{-6} M_{\odot} \mathrm{yr}^{-1}$ and 


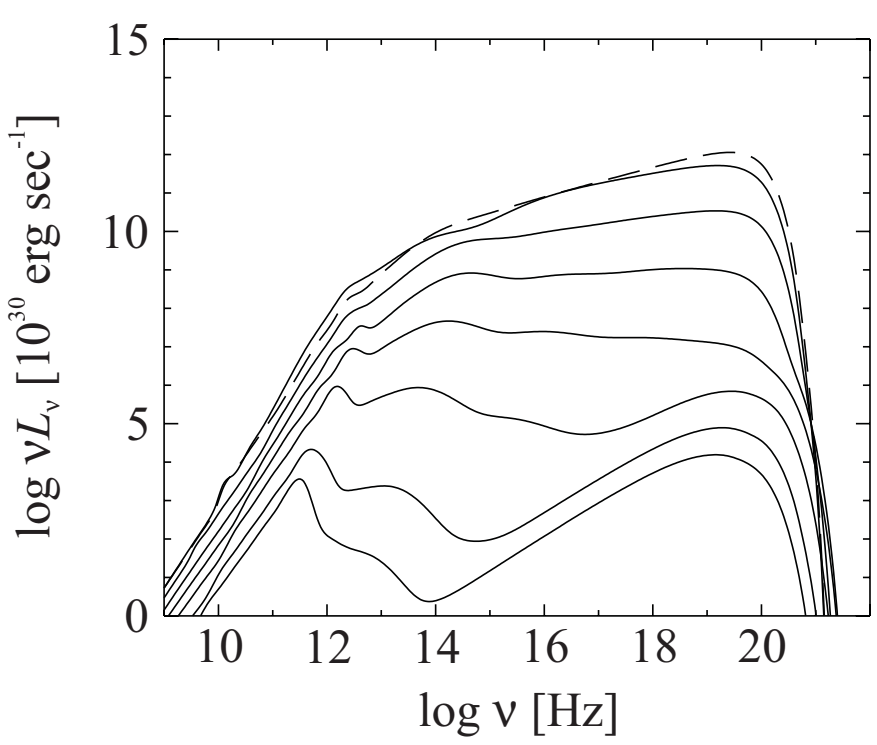

Fig. 1. Spectral energy distribution (SED) for ADAF models with a black hole mass of $2.6 \times 10^{6} M_{\odot}$. The mass accretion rates are $\dot{M}=\left(10^{-6}, 2 \times 10^{-6}, 5 \times 10^{-6}, 10^{-5}, 2 \times 10^{-5}, 5 \times\right.$ $\left.10^{-5}, 10^{-4}, 1.27 \times 10^{-4}\right) M_{\odot} \mathrm{yr}^{-1}$. The scale-free accretion rate for this $\mathrm{BH}$ is $\dot{m}=17.33\left[\dot{M} /\left(M_{\odot} \mathrm{yr}^{-1}\right)\right]$. The spectral energy flux increases for increasing mass accretion rate with an exception at $\dot{M}=1.27 \times 10^{-4}$, which is plotted with a dashed curve.

an efficiency of $\eta=2.2 \times 10^{-8}$. The sub-mm luminosity of Sgr $\mathrm{A}^{*}$ is about 30 times larger than the X-ray flux and makes Sgr A* a unique object. We will discuss a specific ADAF model for Sgr A* in Sect. 3. The Bondi flow faces at least two problems: it does not allow for any possible angular momentum of the inflow and does not include magnetic fields, which lead to synchrotron emission at radio frequencies and synchrotron self-compton cooling. Both can be accounted for in ADAF models. They provide a reasonable explanation for the spectral energy distribution (SED) of Sgr $\mathrm{A}^{*}$ with a mass accretion of $\approx 10^{-6} M_{\odot} \mathrm{yr}^{-1}$ and a radiative efficiency of $10^{-5}$. Beside the basically unresolved radio core of $\operatorname{Sgr} \mathrm{A}^{*}$, it is not possible to identify a jet in the Galactic Center.

In this paper we will explore the hypothesis, that most of the normal galaxies without substantial AGN activity contain supermassive black holes $M>10^{6} M_{\odot}$ some of which have been active during the quasar phase $0.3<z<5$ ( $z$ being the cosmological redshift) and are quietly accreting in an ADAF mode today. Spectral properties of ADAFs with rather large mass accretion rates are explored in Sect. 2. The total luminosities and spectral energy distributions are of interest for weak AGNs (Ho 1999). We investigate the transition from standard thin disk accretion to ADAFs and vice versa as an upper limit in the mass accretion rate for ADAFs in Sect. 4. The combined consequences for accretion in normal galaxies are discussed in Sect. 5.

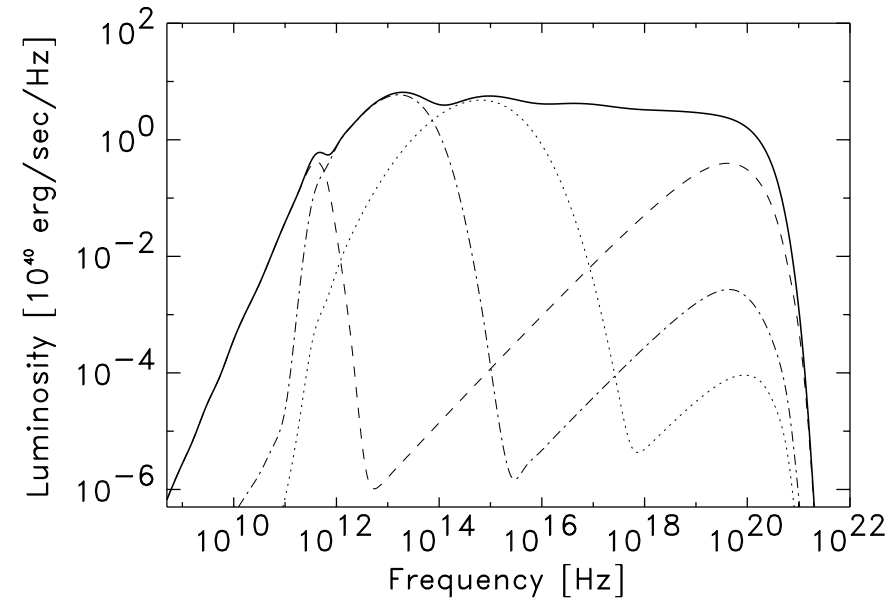

Fig. 2. SED for an ADAF around a $10^{9} M_{\odot}$ black hole. The mass accretion rate is $\dot{m}=3.6 \times 10^{-4}$ corresponding to $8 \times$ $10^{-3} M_{\odot} /$ yr. The seed photon flux is represented as dashed line, the first and second Compton humps are dash-dotted and dotted respectively. Further Comptonization is important but individual humps are smeared out. Only the total SED is shown as solid line. The dependence of the spectral shape on the black hole mass is weak, while the total flux scales with the mass accretion rate according to Eq. (2).

\section{Radiation characteristics of ADAFs}

The calculation of emission spectra from ADAFs are based on self-similar solutions for density, inflow velocity, rotation, and ion temperature presented in Beckert (2000), which are a generalisation of the Narayan \& Yi (1994) solution in the Newtonian limit. Gravitational redshift and the small volume of the general-relativistic region makes it reasonable to extend the solution down to the black hole horizon at $R_{\mathrm{S}}$. Aspects of the Kerr metrics are not considered. The electron temperature is calculated from thermal balance between Coulomb heating by ions, adiabatic compression, advective energy transport, and radiation cooling. The primary radiation mechanisms are Bremsstrahlung and synchrotron emission. Both are local in the sense, that they only depend on the local state of the flow. At large mass accretion rates multiple inverse Compton scattering (IC) of synchrotron photons becomes the dominant cooling mechanism. The photon flux to be scattered is produced at different radii in the flow and is highly anisotropic, which makes it a non-local process. Inverse Compton scattering and the resulting cooling rate modify the density and temperature of the ADAF and are treated iteratively. For calculating the IC radiation, we used a method proposed by Poutanen \& Svensson (1996) for thermal electrons, which we have modified to allow for the quasi-spherical geometry of ADAFs. From the total cooling we take the global radiation efficiency $\varepsilon_{\mathrm{Rad}}=Q^{-} / Q^{+}$to recalculate the selfsimilar ADAF structure and electron temperature. We then seek convergence of the assumed and posteriori calculated $\varepsilon_{\text {Rad }}$ to get globally consistent ADAF models with correct radiation spectra. For the ADAF we assume energy 
equipartition between ions and magnetic field. This determines the synchrotron emission and provides additional pressure to support the flow and lower the adiabatic index below $5 / 3$ to make ADAFs possible at all. For the viscosity we use the standard $\alpha$-prescription with $\alpha=0.1$ well above the critical value of $\alpha_{\text {crit }} \approx 10^{-2}$ for the transition to convection-dominated accretion flows (CDAFs) described by Narayan et al. (2000). No outflows, jets or wind infall as investigated in Beckert (2000) are assumed, even though a wind infall is suggested for the Galactic Center (Melia \& Coker 1999). The radial viscous break due to bulk and shear viscosity is included, but it does not dominate the dynamical state of the flow for $\alpha \leq 0.1$.

From the described model we can construct spectral energy distributions (SED) for different mass accretion rates. The mass of the central $\mathrm{BH}$ has only a weak influence on the SED, which is not included in the scaling of $\dot{M}$ to the Eddington accretion rate, and is not considered here. We assume a mass of $2.6 \times 10^{6} M_{\odot}$ for the SED in Fig. 1, appropriate for the Galactic Center, with a corresponding Eddington limit of $\dot{M}_{\mathrm{Edd}}=0.0577 M_{\odot} \mathrm{yr}^{-1}$ (in this paper we define the Eddington accretion rate with an efficiency $\left.\eta=L_{\mathrm{Edd}} /\left(\dot{M}_{\mathrm{Edd}} c^{2}\right)=0.1\right)$ and use the scale free accretion rate $\dot{m}=\dot{M} / \dot{M}_{\text {Edd }}$ in the following discussion. The spectral luminosity in Fig. 1 scales as the black hole mass, $\nu L_{\nu} \propto M_{\mathrm{BH}}$. For comparision we show in Fig. 2 the SED for an ADAF arround a $10^{9} M_{\odot}$ black hole with $\dot{m}=3.6 \times 10^{-4}$. Figure 5 demonsttrates that the radiation efficiency for this flow is independend of the black hole mass.

The SEDs for $\dot{m}$ between $1.7 \times 10^{-5}$ and $2.2 \times 10^{-3}$ are shown in Fig. 1. The presented model spectra are accurate above $30 \mathrm{GHz}$, and they show that the synchrotron emission rises in flux from $10^{33}$ to $10^{37} \mathrm{erg} \mathrm{s}^{-1}$ and shifts in frequency from $2 \times 10^{11} \mathrm{~Hz}$ to $2 \times 10^{12} \mathrm{~Hz}$ at $\dot{m}=3.5 \times 10^{-4}$ and back to smaller frequency for larger $\dot{m}$. Above $\dot{m}=1.5 \times 10^{-4}$ the Thomson optical depth for synchrotron photons from central regions around $3 R_{\mathrm{S}}$ is significant, and Compton scattering broadens the synchrotron peak and make it less prominent, compared to the IC emission. The dominating peak in the IC part of the spectrum, which can be identified between the synchrotron and the Bremsstrahlung peaks at $10^{19 \ldots 20} \mathrm{~Hz}$, is the second Compton peak of twice scattered synchrotron photons. The synchrotron seed photons are produced in a region closer to the $\mathrm{BH}$ than the Compton scattered radiation. So the seed photon flux for first Compton scattering is anisotropic, and most synchrotron photons are scattered back into a high density and high temperature region with the largest optical depth. The second Compton peak is therefore the dominant one, and the asymmetry between even and odd scattering order decreases thereafter, because the photon field to be scattered becomes more and more isotropic. The SED becomes flat or inverted due to multiple IC scattering above $10^{14} \mathrm{~Hz}$ for $\dot{m} \geq 3 \times 10^{-4}$. The Bremsstrahlung peak will only be recognized below $\dot{m}<1.5 \times 10^{-4}$. The peak does not shift very much in frequency as the maximum of electron temperature only

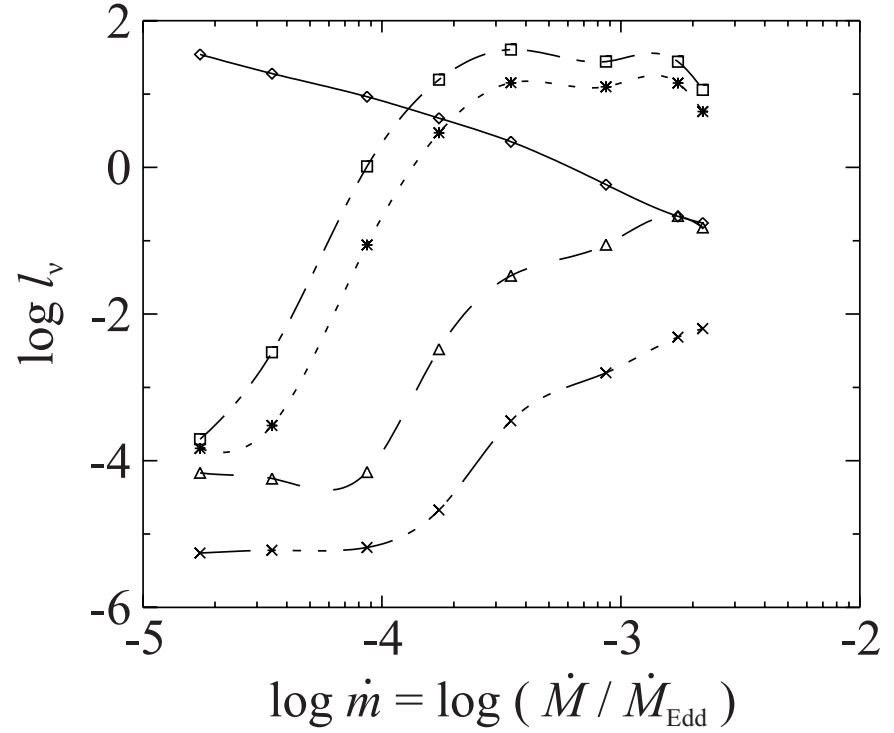

Fig. 3. Normalized flux $l_{\nu}$ (see Eq. (1)) at $86 \mathrm{GHz}$ (solid line), in $K$ (dash-dotted) and $V$ band (dotted), at $1 \mathrm{keV}$ (dashed) and $100 \mathrm{keV}$ ( $3 \times$ dotted-dashed) as a function of mass accretion rate. The models are calculated for a $2.6 \times 10^{6} M_{\odot}$ black hole. The efficiency $\eta$ in Eq. (1) is $10 \%$ and the SED is assumed to peak at $100 \mathrm{keV}$.

varies between $2.5 \times 10^{9} \mathrm{~K}$ and $8 \times 10^{9} \mathrm{~K}$, where the highest $T_{\mathrm{e}}$ are achieved at $\dot{m} \approx 2 \times 10^{-4}$ very close to the horizon and the photons from that radius are significantly redshifted.

One major prediction of ADAF models is the different evolution of observable flux in different frequency bands. This is expressed in the scale-free spectral luminosity $l_{\nu}$. We scale the radiation flux $L_{\nu}$ to the total, frequency integrated luminosity of the specific model from Eq. (2) and define

$l_{\nu}=\frac{L_{\nu}}{\eta \dot{M}_{\mathrm{Edd}} c^{2}}\left[\frac{\dot{m}}{10^{-3}}\right]^{-2.3}\left[\frac{h \nu_{\max }}{100 \mathrm{keV}}\right]$.

We see in Fig. 3 that the importance of synchrotron emission at $86 \mathrm{GHz}$ decreases with rising $\dot{m}$; the X-rays follow the total luminosity at first, and become more important when they are dominated by IC emission above $\dot{m} \geq 2 \times 10^{-4}$. The importance of IC emission is most prominently seen in $K$ and $V$ band. The flux is rapidly rising with $\dot{m}$ and saturates above $\dot{m}=2 \times 10^{-4}$ when IC is dominating the cooling and therefore IC follows the total luminosity. The described spectra show only a weak dependence on the absolute mass of the $\mathrm{BH}$ between $10^{4}$ and $10^{9} M_{\odot}$, for which we have tested the models. Larger $\mathrm{BH}$ masses imply smaller densities in the accretion flow at the same $\dot{m}$. Consequently the electron temperature also becomes smaller due to weaker Coulomb coupling. As the only significant consequence for the SED, the position of the synchrotron peak is anticorrelated with $M_{\mathrm{BH}}$, and it shifts to smaller frequencies for larger $\mathrm{BH}$ masses. 


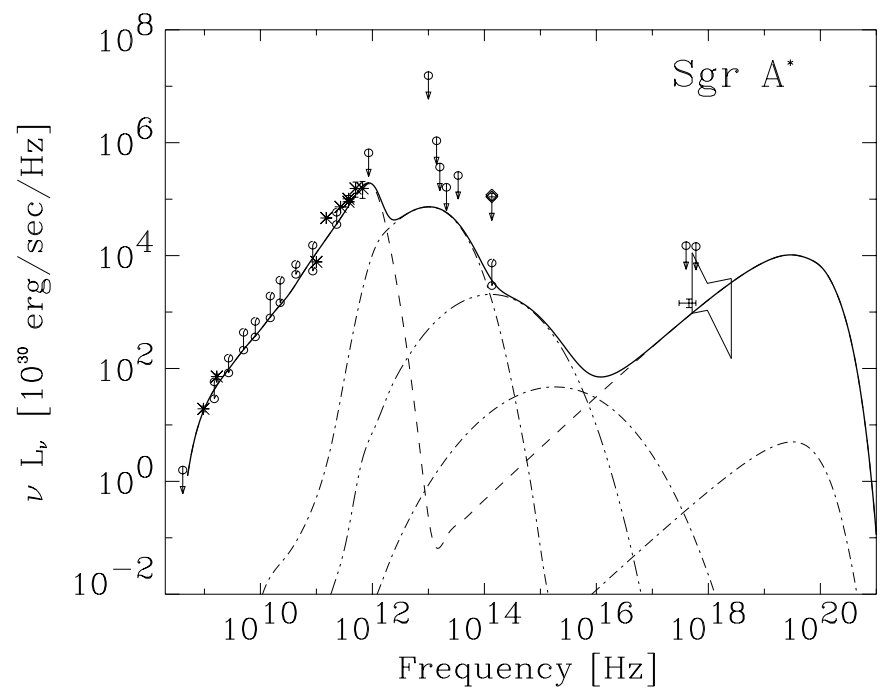

Fig. 4. Spectral energy distribution (SED) for an ADAF model with a wind infall appropriate for Sgr A*. The black hole mass is $2.6 \times 10^{6} M_{\odot}$ and the mass accretion rate at the last stable orbit $\dot{M}=9 \times 10^{-7} M_{\odot} \mathrm{yr}^{-1}$ corresponding to $\dot{m}=1.6 \times 10^{-5}$. This special model is briefly described in Sect. 3. The dashed line shows the synchrotron and bremsstrahlung emission. The first three inverse Compton stages are given as dash-dotted lines. The total spectrum (thick line) has a remarkably flat radio slope $\nu L_{\nu} \propto \nu^{1.3}$ consistent with the observations.

\section{A note on the galactic center source Sgr A*}

The enigmatic radio source Sgr $\mathrm{A}^{*}$ in the Galactic Center is coincident with the center of gravity of an enclosed mass of $2.6 \times 10^{6} M_{\odot}$. It was considered to be one of the test cases for ADAF models (Narayan et al. 1998), but the SED of the source poses three problems for standard ADAFs. (1) The observed radio spectrum is much flatter than predicted, so that only the sub-mm bump (Falcke 1999) is nowadays attributed to the accretion flow. Most of the radio emission at cm-wavelength must then be produced by an outflow or jet (Falcke \& Markoff 2000). (2) The X-ray spectrum as derived from Chandra observations (Baganoff et al. 2002) has a different slope than expected from thermal bremsstrahlung coming from the ADAF. (3) The observed rapid variability in X-rays (Baganoff et al. 2001) restricts the size of the variable emitting region to less than $10 R_{\mathrm{S}}$. The spectrum at high $\mathrm{X}$-ray fluxes is harder than at low flux levels. This can be explained by inverse Compton emission of relativistic electrons in a jet (Markoff et al. 2001) but even a jet has to be powered by an accretion process and bremsstrahlung emission of the accreting gas is unavoidable. In contrast to these recent scenarios, here we present an ADAF-wind infall model, where the gas in the accretion flow is heated by wind infall at all radii (Beckert 2000) with a steeper density profile $\Sigma \propto r^{-1 / 2-\beta}$ than normal ADAFs. The synchrotron emission dominates the SED (Fig. 4) due to a strongly magnetised ADAF $\beta_{P}=P_{\text {Gas }} / P_{\text {total }}<0.5$.
The wind infall is assumed to be strong $\beta=0.24$ and rotates with $\Omega / 2$ of the ADAF. The flow is magnetically dominated with $\beta_{P}=0.35$ and the radiative efficiency $\epsilon=9 \times 10^{-5}$ is larger than in the other models presented in this paper due to the larger electron temperature and the stronger magnetic fields, which leads to increased synchrotron emission. Viscosity is described by an $\alpha$-parametrisation with $\alpha=0.08$, lower than for the other ADAF models in Sect. 2, but convection is still expected to be unimportant (Narayan et al. 2000). This model gives a good fit to the radio spectrum but the problem with the $\mathrm{X}$-ray observation persists.

\section{Limits on the mass accretion rate}

The luminosity of the synchrotron peak relative to Bremsstrahlung and IC emission depends only on $\dot{m}$, and it is unaffected by the absolute mass of the BH. The global radiative efficiency $\varepsilon_{\mathrm{Rad}}$, defined in the previous section, gives the cooling rate $Q^{-}$with respect to the viscous heating rate. The heating rate itself depends on $\varepsilon_{\text {Rad }}$ in the ADAF model, and it is therefore not useful for comparing different models. In describing the efficiency of an accretion flow, it is more reasonable to use $\varepsilon=Q^{-} /\left(\eta \dot{M} c^{2}\right)$. In the following we assume a standard efficiency of $\eta=0.1$. The quantity $\varepsilon$ gives the ratio between the actual luminosity of the accretion flow and the value expected for a thin and effectively radiating accretion disk at the same mass accretion rate. The radiative efficiency $\varepsilon$ is shown for our model calculations in Fig. 5. As described in the previous section, different emission mechanisms dominate at different accretion rates, and we do not expect $\varepsilon$ to follow a simple power law in $\dot{m}$. The fit

$\varepsilon=(340 \dot{m})^{2.2}$

therefore does not represent a theoretically derived physical law, and has to be taken with caution when used outside the scope of the calculated models. Nonetheless the results allow us to conclude that no consistent ADAF models with mass accretion rates larger than $\dot{m}_{\text {crit }}=2.95 \times 10^{-3}$ are feasible. The reason is that IC emission increases rapidly with electron temperature and density. For increasing mass accretion rates both density and surface density increase linearly as long as the accretion velocity stays the same (this is true for ADAFs with $\varepsilon \leq 0.3$, which are consequently advection-dominated). The maximum $T_{\mathrm{e}}$ in the flow depends only weakly on $\dot{m}$ for IC dominated cooling. The Thomson optical depth is

$\tau=\frac{3 \dot{m}}{\eta \alpha} \sqrt{\frac{R_{\mathrm{S}}}{2 r}}$

and the mean energy which a photon gains from one scattering is $2 \gamma^{2} h \nu \approx 6.5 h \nu$. The seed photons have $\nu \approx$ $10^{12} \mathrm{~Hz}$, and upscattering stops if $\left(2 \gamma^{2}\right)^{n}=\gamma m_{\mathrm{e}} c^{2} /(h \nu)$, which results in photons gaining energy in up to $n \approx 10$ consecutive scatterings. Starting from a synchrotron luminosity $L_{\mathrm{s}}$ the IC luminosity will be $L_{\mathrm{IC}} \approx\left(2 \gamma^{2} \tau\right)^{n} L_{\mathrm{s}}$, 


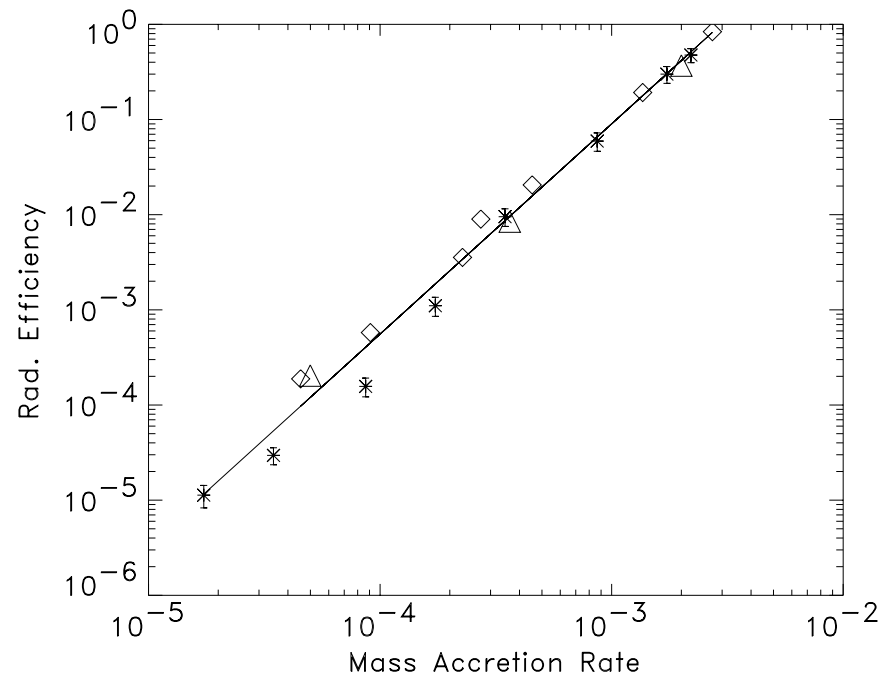

Fig. 5. Radiation efficiency $\varepsilon$ as a function of mass accretion rate $\dot{m}$ with efficiency $\eta=0.1$ to compare with the expectation for efficient radiation cooling. Model calculations are made for a $2.62 \times 10^{6} M_{\odot}$ black hole (symbol: stars), for $10^{8} M_{\odot}$ (diamonds), and for a $10^{9} M_{\odot}$ (triangles) black hole. The estimated errors for the numerical convergence of assumed and posteriori derived $\varepsilon$ are given for the $2.6 \times 10^{6} M_{\odot}$ black hole. The data are fitted with Eq. (2).

if $2 \gamma^{2} \tau>1$, and the spectrum will be flat for $2 \gamma^{2} \tau=1$. In the case of $\eta=\alpha=0.1$ and $r \sim 3 R_{\mathrm{S}}$ this criterion is fulfilled, if $\dot{m}=1.3 \times 10^{-3}$. This is a reasonable order of magnitude estimate, when compared to the model spectra described above. With a constant synchrotron luminosity of $5 \times 10^{-7} L_{\mathrm{Edd}}$, we find in the IC dominated regime

$$
L_{\mathrm{IC}}=5 \times 10^{-7}\left(8 \frac{\dot{m}}{\eta \alpha}\right)^{n} L_{\mathrm{Edd}} .
$$

An upper limit for the mass accretion rate is

$$
\dot{m}_{\mathrm{c}}=0.53 \eta \alpha
$$

which is in rough agreement with the upper limit found by fitting the total luminosity in Eq. (2). It must be noted that this limit $\dot{m}_{\mathrm{c}}$ has a different $\alpha$-dependence than other limits $\dot{m}_{\text {crit }}=\xi \alpha^{2}$ (Narayan 1996; Esin et al. 1997) which give a wide range for $\xi$ between 0.1 and 1.3 depending on details of the models.

Another local criterion for the existence of ADAFs is given by the imbalance of Bremsstrahlung cooling and viscous heating. At large radii the electron cooling rate, which is coupled to the ion heating rate by the radiation efficiency, decreases faster than Coulomb coupling between thermal ions and electrons. The electron temperature is therefore close to the ion temperature at these radii. The ions are close to the viral temperature as long as the radiative efficiency is significantly smaller than 1 , which is the case for all calculated models here. In the region where $T_{\mathrm{e}}$ and $T_{\mathrm{ion}}$ are equal, the Bremsstrahlung cooling, which dominates at large radii, decreases as $r^{-5 / 2}$, while the viscous heating falls off as $r^{-3}$. At a critical outer radius, the radiative efficiency is 1 and no ADAF is possible at larger radii. This outer radius is found to be

$R_{\text {out }}=5 \times 10^{2} \alpha^{4} \dot{m}^{-1} R_{\mathrm{S}}$,

which is valid for $\alpha \leq 0.1$ and means that for $\dot{m}>0.13$ Bremsstrahlung from an ADAF is more efficient than the viscous heating outside the marginally stable orbit at $R=$ $3 R_{\mathrm{S}}$. From studies of the Galactic Center we know that no standard thin accretion disk exists within $10^{5} R_{\mathrm{S}}$ and the mass accretion rate from spectral fitting of an ADAF model gives $\dot{m} \approx 1.6 \times 10^{-5}$. From Eq. (6) this results in a lower limit estimate of $\alpha>0.01$.

\section{Discussion - consequences for AGN evolution}

We find that the radiation efficiency depends strongly on the mass accretion rate $\left(\propto \dot{m}^{2.3}\right)$ below a certain critical value $\dot{m}_{\text {crit }}$ of the mass accretion rate (in units of its Eddington value). Above $\dot{m}_{\text {crit }}, \varepsilon=1$. In other words, below the critical mass accretion rate, the efficiency decreases so rapidly that one can discern two regimes:

$\varepsilon=\left\{\begin{array}{l}1 \text { for } \dot{m}>\dot{m}_{\text {crit }} \\ 0 \text { for } \dot{m}<\dot{m}_{\text {crit }}\end{array}\right.$,

with only a small transition zone in $\dot{m}$ below $\dot{m}_{\text {crit }}$ where $\varepsilon$ differs significantly from 0 . For the purpose of our present discussion, however, the approximation of Eq. (7) suffices.

Furthermore, our numerical models show that the relation outlined by Eq. (7) holds for the entire range of $M_{\mathrm{BH}}$ investigated. In the following we will use the value $\dot{m}_{\text {crit }}=0.003$ as derived by interpolating our numerical models (Eq. (2)).

\subsection{The onset of nuclear activity}

The existence of $\dot{m}_{\text {crit }}$ with the properties discussed above translates into a fairly sharp transition between an active and an inactive state of a galaxy. As soon as $\dot{m}$ falls below $\dot{m}_{\text {crit }}$ the radiation efficiency of the accretion decreases dramatically. In other words, already a relatively small change in the mass flow rate around $\dot{m}_{\text {crit }}$ suffices to "switch off" an AGN, and vice versa. The difference between a normal and an active galaxy is then not due to a difference in $\dot{m}$ which is as large as the difference in luminosities between the two classes. A much more important reason is the steep decline in the radiation efficiency for the accretion rates below which the disk turns advectiondominated.

Our numerical models predict $\dot{m}_{\text {crit }} \sim 0.003$. This is in good agreement with observations (e.g., Peterson \& Wandel 2000, who find AGN only in the luminosity range between $\sim 10^{-3}$ and 1 of the Eddington luminosity, or equivalently - the Eddington mass accretion rate). In our interpretation the lack of galactic nuclei below $10^{-3} L_{\text {Edd }}$ is not due to a lack of galaxies with mass accretion rates below $10^{-3} \dot{M}_{\mathrm{Edd}}$ but rather due to the steep decline of radiation efficiency below this critical value. 


\subsection{The general properties of the evolution of AGN and of their black holes}

For a supermassive black hole of mass $M_{\mathrm{BH}}$, one can give an average accretion rate $\overline{\dot{M}}$ over its age $\tau_{\mathrm{BH}}$ of

$\overline{\dot{M}} \leq \frac{M_{\mathrm{BH}}}{\tau_{\mathrm{BH}}}$

We can only give an upper limit of $\overline{\dot{M}}$, because there is the possibility of a non-negligible seed mass of the black hole which is not due to this type of accretion process.

If, in addition, we assume, that the age of the $\mathrm{BH}$ is not very much shorter than the age of its host galaxy and thus the Hubble time (at the location of the black hole), $\tau_{\mathrm{H}}$, we can write

$\overline{\dot{M}} \leq \frac{M_{\mathrm{BH}}}{\tau_{\mathrm{H}}}$.

In the following, we express the time in units of $10^{10} \mathrm{yr}$, and the mass flow rates in units of the Eddington rate. We introduce the abbreviations $\dot{m}=\dot{M} / \dot{M}_{\text {Edd }}$, and $\tau_{10}=$ $\tau / 10^{10} \mathrm{yrs}$, and get an average mass accretion rate of

$\overline{\dot{m}} \leq 0.04 \eta \frac{1}{\tau_{10}}$.

In terms of the Eddington accretion rate, the average accretion rate $\overline{\dot{m}}$ is independent of the accreting black hole's mass. For a constant accretion efficiency $\eta$, it is $\overline{\dot{m}} \propto t_{\mathrm{BH}}^{-1}$ a function of the age of the black hole, or - for that matter the Universe. This leads to two interesting consequences:

- in terms of the Eddington rate, the accretion rate declines as the black hole, the galaxy, and the Universe as a whole evolve;

- the present-day average accretion rate is around $10^{-3}$ of its Eddington value.

The present-day average accretion rate, $\overline{\dot{m}}_{(0)}$ is fairly close to the critical accretion rate $\dot{m}_{\text {crit }} \sim 0.003$ given by $\varepsilon=1$ (Eq. (2)). For $\dot{m}>\dot{m}_{\text {crit }}$ the radiation efficiency is unity, while for smaller $\dot{m}$ it decreases sharply $\propto m^{2.3}$. This means that relatively small changes in the momentary accretion rate are capable of transferring a galaxy from an inactive to an active state and vice versa. In the course of the further evolution of the Universe, it will become harder and harder for galaxies to turn active as the average accretion rate (in Eddington units) becomes smaller and smaller. This is compounded with and strengthened by a diminishing supply of material available for accretion, i.e., by an additional decrease of the absolute value of the average accretion rate. Extrapolating back to earlier cosmological epochs, we find (because of smaller $\tau_{\mathrm{BH}}$ and higher $\overline{\dot{m}}$ ) that the likelihood for a galaxy to be active was higher on two grounds: (1) The larger $\overline{\dot{m}}$ the smaller a fluctuation suffices to turn a galaxy active. (2) The further we go back in the evolution of the Universe, the larger was the supply of gas available for accretion.

\subsection{Constraints on the AGN duty cycle}

As discussed above, the mass $M_{\mathrm{BH}}$ and age $\tau_{\mathrm{BH}}$ of a black hole define an upper limit for its average mass accretion rate. During phases of activity, the mass flow rate must be larger than $\overline{\dot{m}}$. Let us - for the purpose of a crude estimate - assume that we have only two states, namely the $A G N$ phase, characterized by a mass flow rate $\dot{m}_{\mathrm{AGN}}>\dot{m}_{\text {crit }}$ lasting for a period of time of $\theta \tau_{\mathrm{H}}$, and a normal galaxy phase for which the mass flow rate $\dot{m}_{\text {normal }}<\dot{m}_{\text {crit }}$ is correspondingly smaller so as to maintain the average value $\overline{\dot{m}}$. Let us, moreover, assume ${ }^{1}$ that $(1-\theta) \dot{m}_{\text {normal }} \ll \theta \dot{m}_{\mathrm{AGN}}$, then we get for the duty cycle

$\theta=\frac{\overline{\dot{m}}-\dot{m}_{\text {normal }}}{\dot{m}_{\mathrm{AGN}}-\dot{m}_{\mathrm{normal}}} \geq \frac{\overline{\dot{m}}}{2 \dot{m}_{\mathrm{AGN}}}$

$\dot{m}_{\mathrm{AGN}} \geq \dot{m}_{\text {crit }}$ needs to be fulfilled for an activity phase to occur at all. The stronger the activity of a galaxy, the shorter the time it can be maintained. For instance, for an AGN operating at its Eddington limit, i.e., at $\dot{m}_{\mathrm{AGN}}=1$ this means that it cannot stay at this level of activity integrated over all individual phases of activity - for longer than a fraction $\theta$ of its entire evolution, i.e., some $10^{7} \mathrm{yr}$ in the present-day Universe. A super-Eddington activity level can be maintained only for an even shorter period of time.

Derivation of a more detailed luminosity evolution of an AGN sample requires, however, a treatment more detailed than the above order-of-magnitude estimates. In particular it has to be investigated whether real-world galaxies can maintain a sufficiently high supply of matter for the accretion process over long enough a period of time. This then involves, for instance, questions about the accretion time scales and the long-term development of mass reservoirs. This topic, however, is beyond the scope of the present paper and will be addressed separately (Duschl \& Strittmatter, in prep.)

\section{Conclusions}

We have shown that advection-dominated accretion flows into black holes display changing spectral energy distributions for different mass accretion rates. At very low mass accretion rates below $\dot{M}=5 \times 10^{-5} \dot{M}_{\text {Edd }}$ synchrotron emission and Bremsstrahlung dominate the SED. Above $\dot{M}=2 \times 10^{-4} \dot{M}_{\text {Edd }}$ the inverse Compton radiation from synchrotron seed photons produce flat to inverted SEDs from the radio to X-ray bands. Multiple inverse Compton scattering is the most relevant cooling process for mildly relativistic electrons in two-temperature ADAFs, whenever the actual radiation efficiency in Eq. (2) is larger than 0.07 or the total luminosity larger than $4 \times 10^{-7} L_{\text {Edd }}$. The rapidly increasing cooling efficiency of the inverse

\footnotetext{
1 This assumption is not necessary for activity to set in, as we have seen in the previous section. For the following, however, it is a handy assumption which does not influence the results of our order-of-magnitude estimates.
} 
Compton process sets an upper limit for the mass accretion rate of $\dot{m}_{\text {crit }}=3 \times 10^{-3}$ for ADAFs. At larger mass accretion rates radiative cooling balances the local viscous heating and advective energy transport is unimportant. The resulting flows are less hot, disk-like, and therefore much denser than ADAFs. Below the critical accretion rate, the radiation efficiency declines rapidly $\varepsilon \propto \dot{m}^{2.3}$ and the total luminosity is

$$
L_{\mathrm{ADAF}}=3.45 \times 10^{-3}(\dot{m} / 0.003)^{3.3} L_{\mathrm{Edd}} .
$$

In addition to the changing spectral behavior, the combination of a transition value between ADAFs and normal accretion disks $\left(\dot{m}_{\text {crit }} \sim 3 \times 10^{-3}\right)$, and the very steep decline of the ADAF radiation efficiency below this transition value towards smaller accretion rates $\left(\varepsilon \propto \dot{m}^{2.3}\right)$ leads to an apparent dichotomy in what one observes: Above $\dot{m}_{\text {crit }}$, one will recognize the sources as AGN; while below $\dot{m}_{\text {crit }}$, practically no nuclear activity is observable. In other words, accretion into a super-massive black hole should be prominently visible only at mass flow rates above $\dot{m}_{\text {crit }}$, which is in good agreement with observed AGN distributions. The major difference between AGN and normal galaxies is not so much a difference in mass flow rate - at least not by as much as the difference in luminosities may make us think - but rather one in the radiative efficiency.

At the same time, this means that - at accretion rates around $\dot{m}_{\text {crit }}$ - small changes in the mass flow rate are sufficient to cause a strong difference in radiation efficiency and thus nuclear luminosity. In other words, the crossing of $\dot{m}_{\text {crit }}$ acts almost like a switch which turns AGNs on and off.

Finally, the combination of the black holes' masses and the mass accretion rate allowed us to put constraints on the duty cycle of AGN. It turned out that the most active AGN can maintain this level of activity only for rather short time scales (of the order of some $10^{7}$ years).

Acknowledgements. We wish to thank the referee, Dr. Suzy Collin, for her very helpful report on this paper. This work was in part supported by the Deutsche Forschungsgemeinschaft, $D F G$, through grant SFB439/C2.

\section{References}

Beckert, T. 2000, ApJ, 539, 223

Baganoff, F. K., Bautz, M. W., Brandt, W. N., et al. 2001, Nature, 413, 45

Baganoff, F. K., Maeda, Y., Morris, M., et al. 2002, ApJ, submitted [astro-ph/0102151]

Esin, A. A., McClintock, J. E., \& Narayan, R. 1997, ApJ, 489, 865

Falcke, H. 1999, in The Central Parsecs of the Galaxy, ASP Conf. Ser., 186, 148

Falcke, H., \& Markoff, S. 2000, A\&A, 362, 113

Falcke, H., Nagar, N. M., Wilson, A. S., \& Ulvestad, J. S. 2000, ApJ, 542, 197

Frank, J., King, A. R., \& Raine, D. J. 1992, Accretion Power in Astrophysics 2nd ed. (Cambridge University Press, Cambridge, UK)

Gammie, C. F., Narayan, R., \& Blandford, R. 1999, ApJ, 516, 177

Gebhardt, K., Bender, R., Bower, D., et al. 2000, ApJ, 539, L13

Genzel, R., Eckart, A., Ott, T., \& Eisenhauer, F. 1997, MNRAS, 291, 219

Ho, L. C. 1999, ApJ, 516, 672

Laor, A. 2000, ApJ, 543, L111

Markoff, S., Falcke, H., Yuan, F., \& Biermann, P. L. 2001, A\&A, 379, L13

Melia, F., \& Coker, R. 1999, ApJ, 511, 750

Mundell, C. G., Wilson, A. S., Ulvestad, J. S., \& Roy, A. L. 2000, ApJ, 529, 816

Narayan, R., \& Yi, I. 1994, ApJ, 428, L13

Narayan, R. 1996, ApJ, 462, 136

Narayan, R., Mahadevan, R., Grindlay, J. E., Popham, R. G., \& Gammie, C. 1998, ApJ, 492, 554

Narayan, R., Igumenshchev, I. V., \& Abramowicz, M. A. 2000, ApJ, 539, 798

Peterson, B. M., \& Wandel, A. 2000, ApJ, 540, L13

Poutanen, J., \& Svensson, R. 1996, ApJ, 470, 249

Ulvestad, J. S., Wrobel, J. M., Roy, A. L., et al. 1999, ApJ, $517, \mathrm{~L} 81$ 